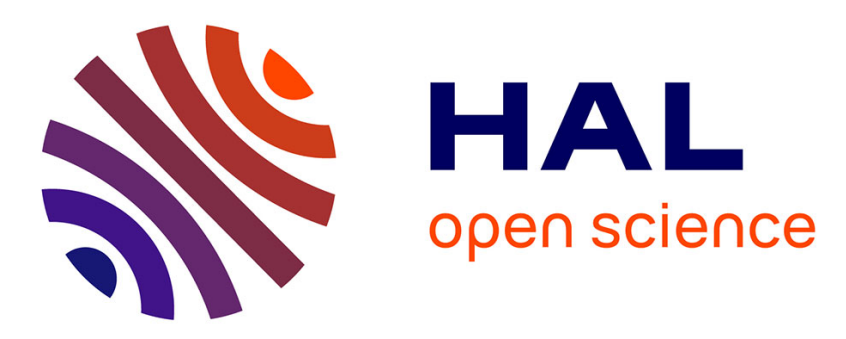

\title{
Behaviour of ambient temperature on daily basis in Italian climate
}

\author{
V. Cuomo, F. Fontana, C. Serio
}

\section{To cite this version:}

V. Cuomo, F. Fontana, C. Serio. Behaviour of ambient temperature on daily basis in Italian climate. Revue de Physique Appliquée, 1986, 21 (3), pp.211-218. 10.1051/rphysap:01986002103021100 . jpa00245433

\section{HAL Id: jpa-00245433 https://hal.science/jpa-00245433}

Submitted on 1 Jan 1986

HAL is a multi-disciplinary open access archive for the deposit and dissemination of scientific research documents, whether they are published or not. The documents may come from teaching and research institutions in France or abroad, or from public or private research centers.
L'archive ouverte pluridisciplinaire HAL, est destinée au dépôt et à la diffusion de documents scientifiques de niveau recherche, publiés ou non, émanant des établissements d'enseignement et de recherche français ou étrangers, des laboratoires publics ou privés. 
Classification

Physics Abstracts

92.60

\title{
Behaviour of ambient temperature on daily basis in Italian climate
}

\author{
V. Cuomo, F. Fontana and C. Serio \\ Dipartimento di Fisica Nucleare, Struttura della Materia e Fisica Applicata, \\ Universita' di Napoli, 80125 Napoli, Italy
}

(Reçu le 8 août 1985, révisé le 14 novembre, accepté le 15 novembre 1985)

\begin{abstract}
Résumé. - Les auteurs ont analysé les séries temporelles sur 20 ans des données de température moyenne journalière relatives à 5 localités italiennes. Ils ont démontré que ces séries temporelles sont bien décrites par la composition de deux composantes périodiques (la première décrivant le comportement périodique de la moyenne et la seconde décrivant le comportement périodique de la déviation standard) et d'une composante stochastique. Ils démontrent, aussi, que la composante stochastique est stationnaire et qu'elle est décrite par un modèle d'autocorrélation linéaire au premier ordre. Le seul harmonique important au point de vue du modèle est celui relatif à la période fondamentale ( 365 jours). En comparant les caractéristiques statistiques des données expérimentales avec celles des données obtenues par l'application du modèle ils ont examiné ses performances.
\end{abstract}

\begin{abstract}
The authors analyse the daily ambient temperature time series in five Italian stations for twenty years (1958-1977). It is shown that these time series are well described mathematically as composite series of two periodic components (one in the mean and the other in the standard deviation) and one stochastic component. Furthermore the stochastic component is stationary and follows the first order linear autocorrelation model of dependence. The only significant harmonic of the periodic components is the one relative to the basic period ( 365 days). Performance of the model is tested by comparing the statistics of generated and experimental data.
\end{abstract}

\section{List of symbols.}

$A_{j}, B_{j}: j$-th Fourier coefficients (series of the daily mean values $(\mu(t))$ of the daily ambient temperature).

$D_{j}, E_{j}: j$-th Fourier coefficients (series of the daily standard deviation values $(\sigma(t))$ of the daily ambient temperature).

$f_{j} \quad$ : frequency of the $j$-th harmonic.

$K_{\mu} \quad$ : number of significant harmonics $(\mu(t)$ series).

$K_{\sigma} \quad$ : number of significant harmonics $(\sigma(t)$ series).

$n \quad$ : number of years in our analysis $(n=20)$.

$R \quad$ : linear correlation coefficient betwen the series $\mu(t)$ and $\sigma(t)$.

$t \quad: t$-th day of the year $(t=1, \ldots, 365)$.

$T(i, t):$ daily ambient temperature $: i$-th year, $t$-th day.

$X(i, t)$ : standardized value of $T(i, t)$; see equation (4).

$Y(i, t)$ : filtered value of $X(i, t)$; see equation (5).

$\mu(t)$ : daily means of $T(i, t)$; see equation (2).

$\langle\mu\rangle$ : overall mean value of $T(i, t)$; see equation (11).

$\rho \quad$ : first autocorrelation coefficient.

$\sigma(t)$ : daily standard deviations of $T(i, t)$; see equation (3).

$\sigma_{T}^{2} \quad$ : overall variance of the time series $T(i, t)$. $\langle\sigma\rangle$ : mean of the series $\sigma(t)$; see equation (10).

$\omega \quad$ : basic period of the series $(\omega=365)$.

A « chapeau " $(-)$ on parameters indicates estimated values (for example $\hat{\rho}$ indicates an estimation of the parameter $\rho$ ).

\section{Introduction.}

Stochastic modelling and simulation has been applied in a wide range of meteorological analysis involving temperature, solar irradiance, precipitation and wind data.

In particular this approach is very interesting for many practical applications, such as the design and performances calculations of renewable energy technologies (e.g. solar energy systems), because it allows one to summarize a large amount of data by using only few statistical parameters.

Among the various stochastic models the Markovian chain has been chosen by many authors as simple model to describe and simulate daily weather events. More specifically, the first order Markov chain has been used for modelling daily global solar irradiance 
and daily ambient temperature by several authors [1-10].

In this papers we show that :

a) time series of ambient temperature exhibit non stationarity both in mean and in standard deviation;

b) the first-order Markov process provides a good analytical basis to describe the statistical behaviour of the standardized time series;

c) periodic component of daily mean and standard deviation of ambient temperature can be fitted by Fourier series using only few Fourier coefficients.

Such approach allows one to build up a sequence of generated data reproducing the statistical features of hystorical data in a very satisfactory way and requiring only few parameters.

This study was intended to investigate the statistical accuracy of some simple models to simulate the daily ambient temperature for the Italian climate.

Performances of the proposed model is tested by comparing the statistics of simulated and experimentally observed data.

\section{Description of the model : basic assumptions, equations and definitions.}

For our analysis we have used daily average values of ambient temperature, $T$, measured during twenty years (1958-1977) in 5 meteorological stations of the "Aereonautica Militaire Italiana" [11] (see Fig. 1).

For every examined station the experimental values of $T$ give a 1-day discrete time series (of length 7300)

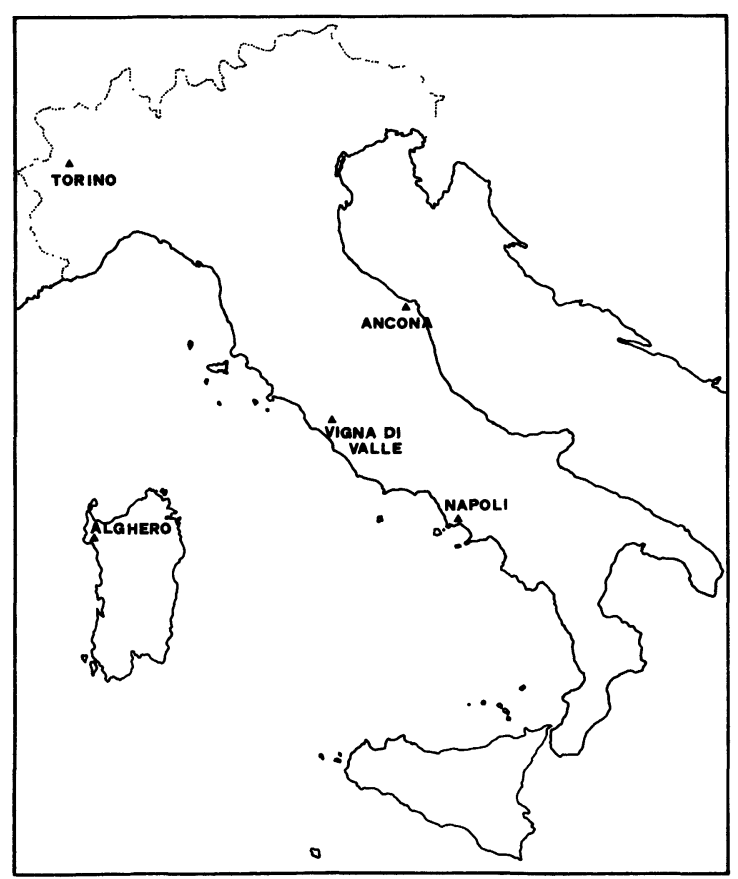

Fig. 1. - Map of the meteorological stations whose data have been analysed. that we can regard as a sample or a realization of a stochastic process. Then in order to detect and consequently eliminate trends in the process, it is convenient to divide the original sample into $n$ sub-samples each one of length $\omega$, where $\omega$ is a some suitable basic period $[12,13]$. In this way the original samples is described by means of a $n \cdot \omega$ matrix :

$$
T(i, t): i=1, \ldots, n ; \quad t=1, \ldots, \omega
$$

with $n \cdot \omega=N$, i.e. the length of the experimental time series ( $N=7300$ in our case) and where :

$\omega$ : is the basic period in the series $T$, expressed as a discrete number;

$n:$ is the number of periods $\omega$ in the finite experimental series $T$.

For daily ambient temperature time series, the year is a basic period : for this reason we assume $\omega=365$ (one year) so that $n$ is the number of years (20 in our analysis) and $n \cdot \omega=7300$ is the length of the sample series.

The yearly trends in the mean and in the standard deviation of the process can be detected computing for each fixed $t$ (day of the year) the estimations $\hat{\mu}(t)$ and $\hat{\sigma}(t)$, respectively of the daily mean and daily standard deviation, according to :

$$
\begin{aligned}
& \hat{\mu}(t)=\frac{1}{n} \sum_{i=1}^{n} T(i, t) \\
& \hat{\sigma}(t)=\frac{1}{n-1} \sum_{i=1}^{n}\left[(T(i, t)-\hat{\mu}(t))^{2}\right]^{1 / 2} .
\end{aligned}
$$

Daily ambient temperature exhibits an obvious trend in the mean; furthermore in the section 3 we show that it exists a seasonal trend in the variance too; in other words $\sigma(t)$ is not a constant along the time series $T$.

In order to eliminate these two seasonal trends of the ambient temperature we use the standardized variable $X$, defined by :

$$
\begin{aligned}
X(i, t) & =\frac{T(i, t)-\mu(t)}{\sigma(t)} \\
t & =1, \ldots, \omega \\
i & =1, \ldots, n
\end{aligned}
$$

$T(i, t):$ is the experimental values of daily average ambient temperature recorded on the $t$-th day of the $i$-th year;

$\mu(t) \quad:$ is the trend in the mean;

$\sigma(t) \quad:$ is the trend in the standard deviation.

The estimations $\hat{\mu}(t)$ of $\mu(t)$ and $\hat{\sigma}(t)$ of $\sigma(t)$ are given respectively by equations (2) and (3).

In section 4 we use the estimations $\hat{\mu}(t)$ and $\hat{\sigma}(t)$ to build up the residual time series $X(i, t)$.

We show that the variable $X$ is not independent and that its empirical distribution is well described by a standard normal distribution. Furthermore we show 
that the series $X(i, t)$ is well described by a first order Markov chain :

$$
X(i, t)=\rho . X(i, t-1)+Y(i, t)
$$

where :

$\rho \quad:$ is the first autocorrelation coefficient;

$Y(i, t):$ is an independent normal variable with zero mean and $\left(1-\rho^{2}\right)^{1 / 2}$ standard deviation.

When the autocorrelation coefficient and the distribution function of $X$ are known, expression (5) allows one to build the sequence $X(i, t)$ and then using the inverse expression of equation (4), i.e. :

$$
T(i, t)=\hat{\mu}(t)+\hat{\sigma}(t) \cdot X(i, t)
$$

it is possible to calculate simulated values of $T$ with the same statistical properties (i.e. mean, standard deviation and autocovariance) as the historical sequences of data.

However such approach is not suitable for practical applications, since it requires the knowledge of the empirical estimations of the daily means and standard deviation, i.e. 365 estimations $\hat{\mu}(t)$ and 365 estimations $\hat{\sigma}(t)$.

In section 5 we show that the series $\hat{\mu}(t)$ and $\hat{\sigma}(t)$ can be fitted very well by Fourier series. The development in Fourier series allows one to describe mathematically $\mu(t)$ and $\sigma(t)$ as :

$$
\begin{aligned}
& \mu(t)=\langle\mu\rangle+\sum_{j=1}^{K_{\mu}}\left(A_{j} \cdot \cos \left(2 \pi f_{j} t\right)+B_{j} \cdot \sin \left(2 \pi f_{j} t\right)\right) \\
& \sigma(t)=\langle\sigma\rangle+\sum_{j=1}^{K_{\sigma}}\left(D_{j} \cdot \cos \left(2 \pi f_{j} t\right)+B_{j} \cdot \sin \left(2 \pi f_{j} t\right)\right)
\end{aligned}
$$

where :

$\langle\mu\rangle:$ is the mean value of the $\mu(t)$ series;

$\langle\sigma\rangle:$ is the mean value of the $\sigma(t)$ series;

$K_{\mu} \quad$ : is the number of significant harmonics of the periodic component in the mean;

$K_{\sigma} \quad$ : is the number of significant harmonics of the periodic component in the standard deviation;

$f \quad$ : is the frequency of the $j$-th harmonic;

$$
f_{j}=\dot{=} j / \omega \quad\left(j=1, \ldots, K_{\mu} \text { or } j=1, \ldots, K_{\sigma}\right)
$$

and $A_{j}, B_{j}, D_{j}, E_{j}$ are the Fourier coefficients.

The parameters $\langle\mu\rangle$ and $\langle\sigma\rangle$ are respectively estimated by :

$$
\begin{aligned}
& \langle\mu\rangle=\frac{1}{\omega} \sum_{t=1}^{\infty} \hat{\mu}(t) \\
& \langle\sigma\rangle=\frac{1}{\omega} \sum_{t=1}^{\infty} \hat{\sigma}(t) .
\end{aligned}
$$

We want to note that the smoothed estimations of the trends in the mean $(\mu(t))$ and in the standard deviation $(\sigma(t))$ given respectively by equations (7) and (8), might be used as well as the empirical values $\hat{\mu}(t)$ and $\hat{\sigma}(t)$ (Eqs. (2) and (3)) in equation (4) to obtain the reduced variable $X$.

The estimators $\hat{\mu}(t)$ and $\hat{\sigma}^{2}(t)$ defined by equations (2) and (3) are affected by a statistical error of order $n^{-1 / 2}(n=20$ in our case), namely [14] :

$$
\begin{aligned}
\operatorname{var}(\hat{\mu}(t)) & =\frac{\sigma^{2}(t)}{n} \\
\operatorname{var}\left(\hat{\sigma}^{2}(t)\right) & =\frac{2 \cdot \sigma^{4}(t)}{n-1}+\frac{K_{4}(t)}{n}
\end{aligned}
$$

where var stands for variance and $K_{4}(t)$ is the fourth cumulant of the population. Because of equations (10.1) and (10.2) then larger fluctuations are expected in the time behaviour of $\hat{\sigma}(t)$, than $\hat{\mu}(t)$ (compare Fig. 5 and Fig. 6 in section 6).

However, in our study we use only the empirical values $\hat{\mu}(t)$ and $\hat{\sigma}(t)$ to analyse the statistical properties of the variable $X$ (Sect. 4), as soon as this is done we compute smoothed estimation of both trens in the mean and in the standard deviation (Sect. 5) and use them to generate simulated values of $T$ (Sect. 6).

\section{Variance analysis.}

In order to check statistical compatibility among the daily standard deviations, $\sigma(t)$, we have used the $F$-test for variance comparison which gives significant results also when applied to small samples (20 data samples in our case) [14].

For each examined station the corresponding daily average ambient temperature, $T(i, t)$, gives $\omega$ samples $(\omega=365)$ :

$$
\{T(i, 1) ; i=1, \ldots, n\}, \ldots,\{T(i, \omega) ; i=1, \ldots, n\}
$$

with $n=20$ ( $n$ is the numbers of years of available data), from which the 365 daily standard deviations, $\hat{\sigma}(t)$, can be computed according to equation (3).

The $F$-test is applied directly to these $\omega$ samples. The $F$-test results indicate that the daily standard deviations are statistically different among them, in other words the time series $T(i, t)$ exhibit a seasonal trend in the standard deviation.

For all the examined stations, in table I we show the observed linear correlation coefficient $R$ between the values $\hat{\sigma}(t)$ and $\hat{\mu}(t)$. We want to stress that the observed correlations are statistically significant for all the localities. In fact the $95 \%$ confidence interval (IC) of the statistic $R$ (when the true value $\rho$ of $R$ is $\rho=0)$ is $I C=(-0.104,0.104)$.

The computed correlation coefficients never belong to $I C$, i.e. every observed $R$ is statistically different from zero. These results not only confirms that $\sigma(t)$ is not a constant, but also indicate that the series $\hat{\mu}(t)$ and $\hat{\sigma}(t)$ will show the same significant harmonics (frequencies) when fitted with Fourier series. 
Table I. - Computed values of the linear correlation coefficient $(R)$ between $\hat{\mu}(t)$ and $\hat{\sigma}(t)$. The $95 \%$ confidence interval (IC) of the statistic, when the true value $\rho$ of $R$ is $\rho=0$, is $I C=(-0.104,0.104)$.

\begin{tabular}{|c|c|}
\hline & $R$ \\
\hline Alghero & -0.34 \\
\hline Ancona & -0.39 \\
\hline Napoli & -0.75 \\
\hline Torino & -0.49 \\
\hline
\end{tabular}

Vigna di Valle

$-0.28$

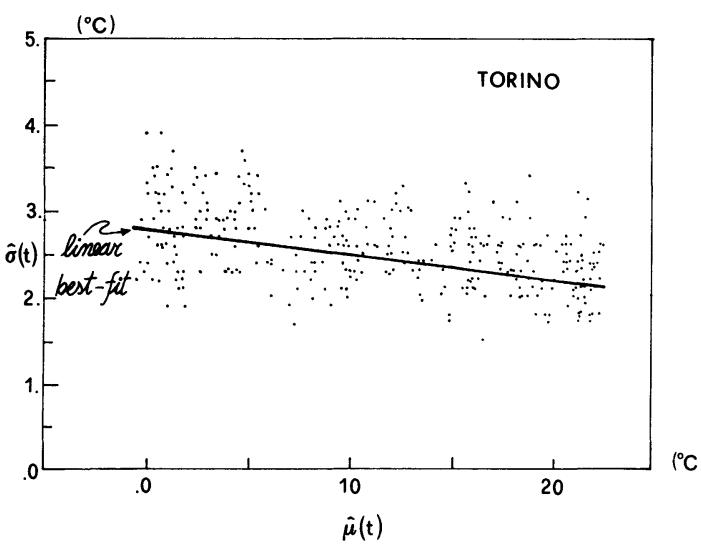

Fig. 2. - Torino station : plot of the daily standard deviations $(\sigma(t)) v s$. the daily means $(\mu(t))$. The values $R$ of the linear correlation coefficient and the extremes of the confidence interval $(I C)$ of the $R$-statistic are shown in table $I$. In all the examined cases the variables are statistically correlated.
Figure 2 shows the points of $\hat{\sigma}(t) v s$. $\hat{\mu}(t)$ with the linear best fit for the Torino station.

\section{Statistical analysis of the residual time series $X(i, t)$.}

Using the experimental data of $T(i, t)$ and the computed values of $\hat{\mu}(t)$ and $\hat{\sigma}(t)$ (Eqs. (2) and (3)) we can obtain the residual time series $X(i, t)$ according to equation (4).

As a first step we check the hypothesis that the residuals computed according to equation (1) are independent by rows and columns, using the ranktest [15].

The rank-test statistic, $r$, is computed across the ensemble of series $\left(\hat{r}_{t} ; t=1, \ldots, \omega\right)$ and along the discrete series $\left(\hat{r}_{i}^{0} ; i=1, \ldots, n\right)$, i.e.

$$
\begin{array}{c|c}
X(1,1), \ldots, X(1, \omega) & \hat{r}_{1}^{0} \\
\ldots \ldots \ldots \ldots \ldots \ldots & \dot{\hat{r}}_{j}^{0} \\
X(n, 1), \ldots, X(n, \omega) & \dot{\hat{r}}_{n}^{0} \\
\hline \hat{r}_{1}, \ldots \hat{r}_{i} \ldots, \hat{r}_{\omega} &
\end{array}
$$

The rank-test results indicate that statistical independence holds well for columns but not for rows where data seem to be strongly autocorrelated. Consequently we suppose that $X(i, t)$ is an autocorrelated variable.

For this reason we check the hypothesis that the residuals can be described by a first order autoregressive model :

$$
X(i, t)=\rho \cdot X(i, t-1)+Y(i, t)
$$

with $\rho$ first autocorrelation coefficient, $Y(i, t)$ independent stochastic variable with zero mean.

Let $X(k)(k=1, \ldots, n \cdot \omega)$ indicates the series obtained ordering the $X(i, t)$ values by year, then an estimation $\hat{\rho}$ of $\rho$ is given by $[12,13,15]$

$$
\hat{\rho}=\frac{\frac{1}{n \cdot \omega} \sum_{k=1}\left((X(k)-\langle X\rangle) \cdot\left(X(k+1)-\left\langle X^{\prime}\right\rangle\right)\right)}{\frac{1}{n \cdot \omega}\left[\sum_{k=1}^{n \cdot \omega-1}\left((X(k)-\langle X\rangle)^{2}\right) \cdot \sum_{k=1}^{n \cdot \omega-1}\left(\left(X(k+1)-\left\langle X^{\prime}\right\rangle\right)^{2}\right)\right]^{1 / 2}}
$$

where :

$$
\begin{aligned}
& \langle X\rangle=\frac{1}{n \cdot \omega-1} \sum_{k=1}^{n \cdot \omega-1}(X(k)) \\
& \left\langle X^{\prime}\right\rangle=\frac{1}{n \cdot \omega-1} \sum_{k=1}^{n \cdot \omega-1}(X(k+1))
\end{aligned}
$$

We note that :

$$
\langle X\rangle \simeq\left\langle X^{\prime}\right\rangle \simeq\langle X(i, t)\rangle=0 .
$$

In table II we show the values of $\hat{\rho}$ obtained for the examined stations. If the model in equation (11) holds, the time series $Y(i, t)$ must be random, i.e. $Y(i, t)$ must be an independent stochastic variable. We compute the values of the time series $Y(i, t)$ from the equation (11), using the estimated values of $\rho$ and the values of $X(i, t)$. As previously done for the time series $X(i, t)$ we check the hypothesis of independence for each one of the 365 columns of the matrix $Y(i, t)$ and for each one of the 20 rows of the matrix $Y(i, t)$ using the rank-test.

In this case the rank-test results confirm, at a significance level of $5 \%$, that the variable $Y$ is uncorrelated. 
Table II. - Estimation of the first autocorrelation coefficient of the time series $X(i, t)$.

\begin{tabular}{|c|c|c|}
\hline Station & $\hat{\rho}$ & $\hat{\sigma}(\hat{\rho})$ \\
\hline Alghero & 0.76 & 0.01 \\
\hline Ancona & 0.77 & 0.01 \\
\hline Napoli & 0.77 & 0.01 \\
\hline Torino & 0.81 & 0.01 \\
\hline Vigna di valle & 0.79 & 0.01 \\
\hline
\end{tabular}

Mean value

0.78

Thus we conclude that the equation (11) holds, i.e. for all the localities $X(i, t)$ is statistically indistinguishable from a linear dependent variable following the first order autoregressive model of dependence.

Moreover the autocorrelation coefficient is independent of time : such results as follows.

We recall that for Markov processes the variance $\sigma^{2}(X)$ of the variable $X$, the variance $\sigma^{2}(Y)$ of the variable $Y$, and the first autocorrelation coefficient $\rho$ are related by :

$$
\sigma^{2}(Y)=\left(1-\rho^{2}\right) \cdot \sigma^{2}(X) .
$$

In our case $\sigma^{2}(X)=1$, so that the problem of systematic variations of $\rho$ along the time series $X(i, t)$ can be investigated checking the homoscedasticity of the time series $Y(i, t)$ (i.e. $\sigma^{2}(i, t)=$ Const.).

So we use again $F$-test for variance comparison [14]; the test is applied to 20 yearly samples of the time series $Y(i, t)$ :

$$
\{Y(1, t) ; t=1, \ldots, \omega\}, \ldots,\{Y(20, t) ; t=1, \ldots, \omega\} .
$$

The results of the $F$-test confirm, at a significance level of $5 \%$, that variations of $\sigma^{2}(Y)$ and therefore of $\rho$, are not statistically meaningful.

In order to investigate eventual dependence on season of the distribution of the variable $X$ we have sub-divided, for each station, the original sample $X(i, t)$ $(i=1, \ldots, n ; t=1, \ldots, \omega)$ in the four sub-samples :

$$
\begin{aligned}
& X_{1}(i, t): i=1, \ldots, n ; t=1, \ldots, 90 \\
& X_{2}(i, t): i=1, \ldots, n ; t=91, \ldots, 181 \\
& X_{3}(i, t): i=1, \ldots, n ; t=182, \ldots, 273 \\
& X_{4}(i, t): i=1, \ldots, n ; t=274, \ldots, 365
\end{aligned}
$$

corresponding respectively to winter (January, February, March), Spring (April, May, June), Summer (July, August, September), and Autumn (October, November, December).

For each stations, histograms were obtained from the samples $X(i, t), X_{1}(i, t), X_{2}(i, t), X_{3}(i, t), X_{4}(i, t)$
(Fig. 3 shows typical results). A Smirnov-Kolmogorov test indicates that differences among them are not statistically significant.

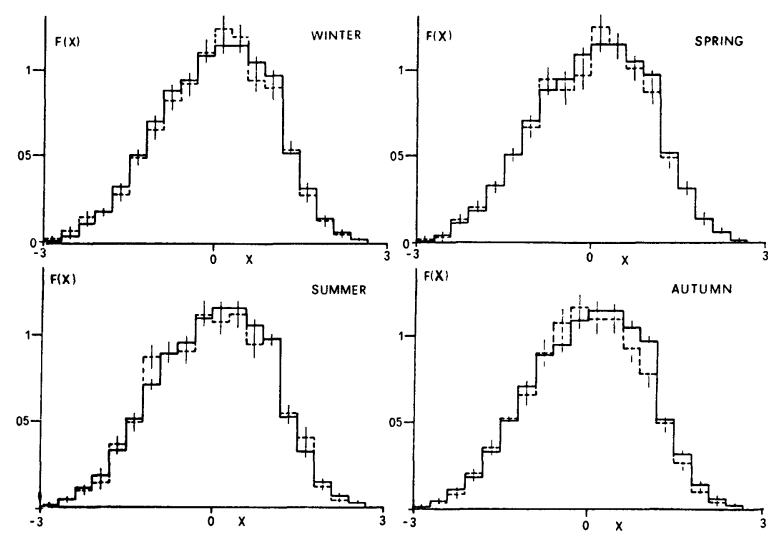

Fig. 3. - Torino station : comparison between the histograms of the variable $X$ : continuous curve refers to the whole time series; dashed curves refer to the values of $X$ related to the four seasons.

Finally, for each stations, the histograms of the variable $X$ was fitted by means of a standardized normal distribution (zero mean and unit standard deviation). Although a $\chi^{2}$-test does not confirm the full statistical compatibility among the standardized distribution and all the empirical distribution functions of the variable $X$, the departure from normality is not serious as it can be seen from figure 4 in which we show the most favourable case $\left(\chi^{2}=34\right)$ and the least favourable case $\left(\chi^{2}=150\right)$.

However, as it will be seen in section 6, the assumption that the stochastic variable $X$ is normal introduces a negligible error when we obtain simulated values of the daily ambient temperature.

\section{Analysis of the series $\mu(t)$ and $\sigma(t)$.}

The model obtained in the previous section for $X(i, t)$ can be used in equation (6) to simulate values of daily ambient temperature. Of course the series $\mu(t)$ and $\sigma(t)$ are needed too. We could use the values of $\hat{\mu}(t)$ and $\hat{\sigma}(t)$ computed on the basis of the historical time series of daily ambient temperature. In practical applications however such approach is very cumbersome since it requires the knowledge of $365 \times 2$ parameters.

In this section we show that Fourier series may be fitted to the computed values of $\hat{\mu}(t)$ and $\hat{\sigma}(t)$ with a few Fourier coefficients.

5.1 THE PERIODIC COMPONENT IN THE MEAN, $\mu(t)$ : ESTIMATION OF THE SIGNIFICANTS HARMONICS. - From each one of the daily ambient temperature time series, $T(i, t)$, we compute the $\omega(\omega=365)$ daily means $\hat{\mu}(t)$, according to equation (2). The Fourier coefficients, 

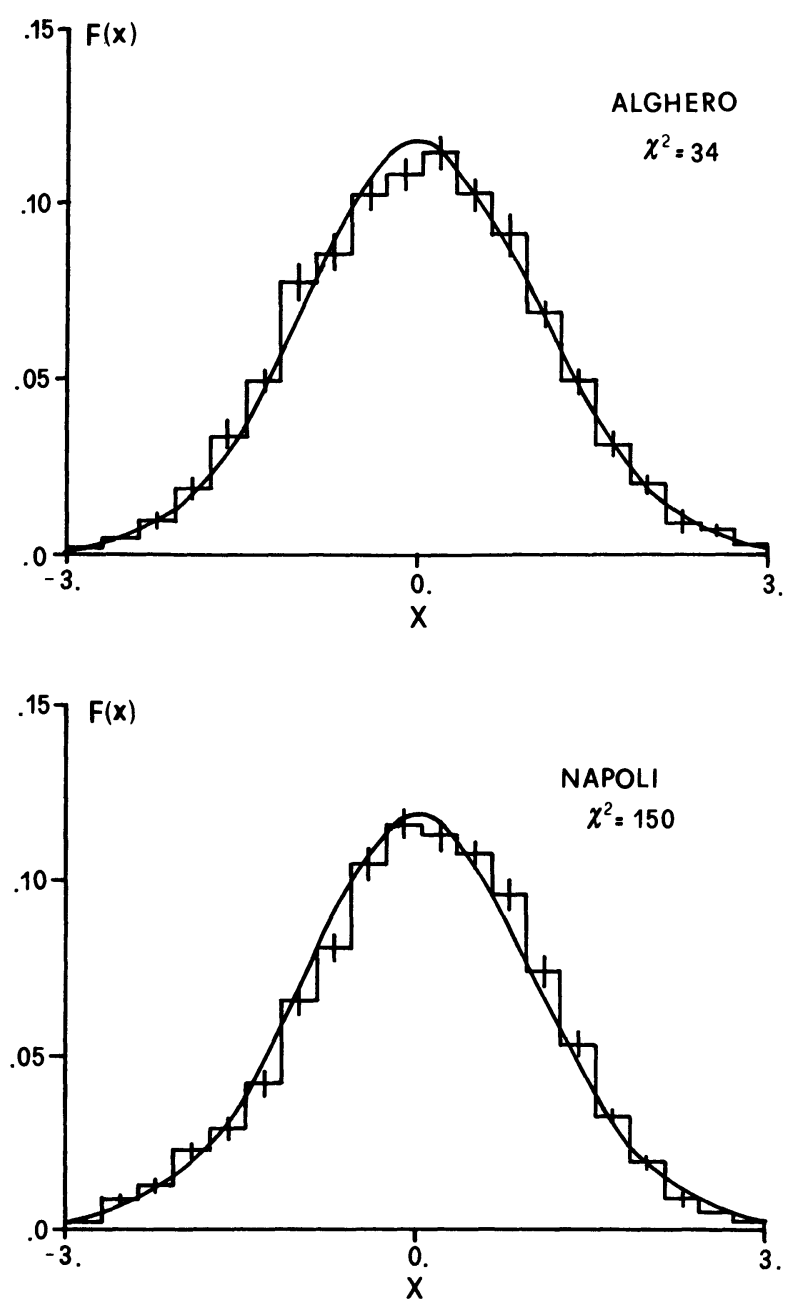

Fig. 4. - Histograms of the variable $X$ (step curve) and its comparison with a standardized normal distribution (continuous curve); the number of freedom degrees of the $\chi^{2}$ variable is indicated in figure 19. In figure we show the most and the least favourable cases.

$A_{j}$ and $B_{j}$, for these series are estimated as usually $[12,13,15]$.

The variance $\hat{\sigma}_{\mathrm{a}}^{2}$, of the series $\hat{\mu}(t)$ is computed according to :

$$
\hat{\sigma}_{\mathrm{a}}^{2}=\frac{1}{\omega} \sum_{t=1}^{\infty}(\hat{\mu}(t)-\langle\mu\rangle)^{2} .
$$

The contribution to the variance $\hat{\sigma}_{\mathrm{a}}^{2}$ for the given $j$-th harmonic is computed according to :

$$
P_{\mathrm{a}}=\frac{A_{j}^{2}+B_{j}^{2}}{2 \cdot \hat{\sigma}_{\mathrm{a}}^{2}} \quad j=1,2, \ldots
$$

In all the cases $P_{\mathrm{a}}$ for the first harmonic is more than $95 \%$.

Therefore the empirical series $\hat{\mu}(t)$ can be described mathematically as follows :

$$
\mu(t)=\langle\mu\rangle+A_{1} \cos \left(\frac{2 \pi \mathrm{t}}{\omega}\right)+B_{1} \sin \left(\frac{2 \pi \mathrm{t}}{\omega}\right) .
$$

For each locality the values of $\langle\mu\rangle, A_{1}, B_{1}, \hat{\sigma}_{\mathrm{a}}^{2}$ and the ratio $P_{\mathrm{a}}$ are given in table III. Figure 5 shows the fitted function, $\mu(t)$, and the 365 computed daily means $\hat{\mu}(t)$ for the Torino station.

Table III. - Computed values of the parameters related to the fitted periodic component in the mean; $\langle\mu\rangle=$ mean value of the 365 daily means $\hat{\mu}(t) ; A_{1}$ and $B_{1}=$ Fourier coefficient of the first harmonic; $P_{\mathrm{a}}=$ contribution of the first harmonic to the variance $\hat{\sigma}_{\mathrm{a}}^{2} ; \hat{\sigma}_{\mathrm{a}}^{2}=\mathrm{va-}$ riance of the 365 daily means $\hat{\mu}(t)$.

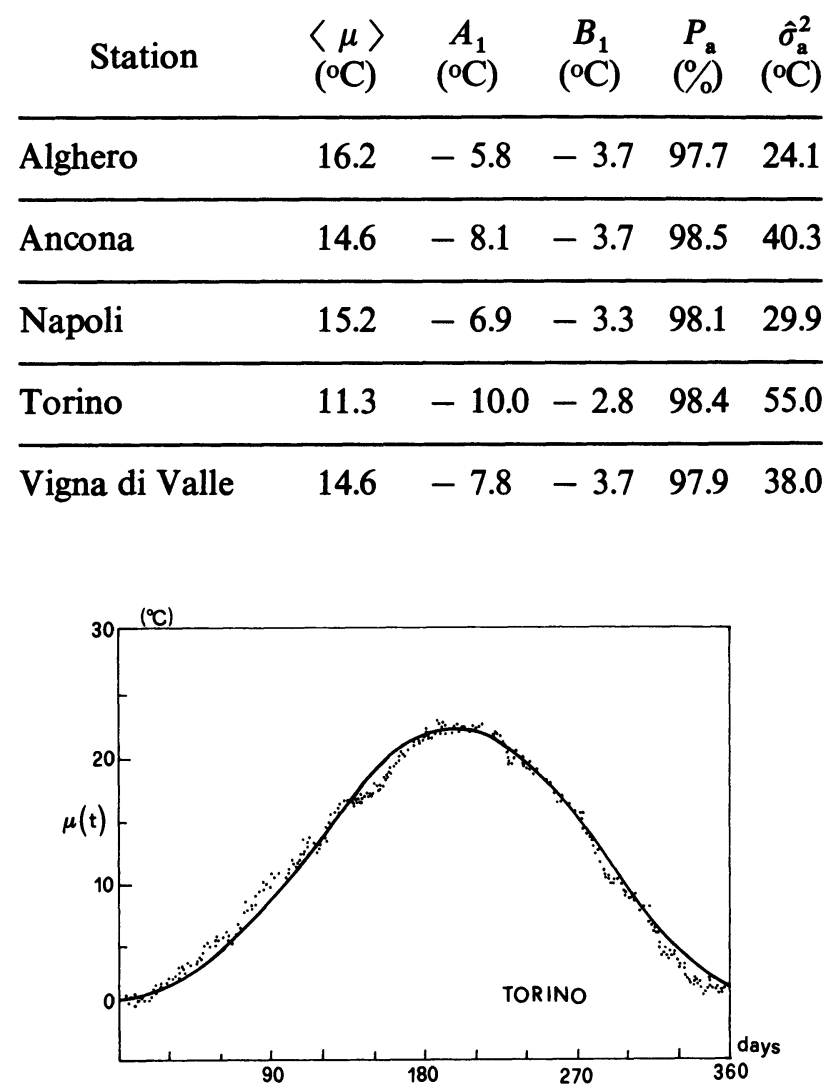

Fig. 5. - Torino station : $\mu(t) v$ s. time (day of the year); continuous curve $=$ fitted function; dotted curve $=$ computed values $\hat{\mu}(t)$.

5.2 THE PERIODIC COMPONENT IN THE STANDARD DEVIATION $\sigma(t)$ : ESTIMATION OF THE SIGNIFICANT HARMONICS. - From each one of the daily ambient temperature time series, $T(i, t)$, we compute the $\omega$ daily standard deviation, $\hat{\sigma}(t), t=1, \ldots, \omega$, according to equation (3). The Fourier coefficients $D_{j}$ and $E_{j}$ for these series are estimated are usually $[12,13,15]$.

In all cases the first harmonic is the more significant. Therefore the empirical series $\hat{\sigma}(t)$ can be described mathematically as :

$$
\sigma(t)=\langle\sigma\rangle+D_{1} \cos \left(\frac{2 \pi t}{\omega}\right)+E_{1} \sin \left(\frac{2 \pi t}{\omega}\right) .
$$

For each locality the values of $\langle\sigma\rangle, D_{1}, E_{1}, \hat{\sigma}_{\mathrm{v}}^{2}$ and 
the ratio $P_{\mathrm{v}}=\frac{D_{1}^{2}+E_{1}^{2}}{2 \cdot \hat{\sigma}_{\mathrm{v}}^{2}}$ are given in table IV; $\hat{\sigma}_{\mathrm{v}}^{2}$ is an estimation of the variance of the series $\hat{\sigma}(\mathrm{t})$, i.e.

$$
\hat{\sigma}_{\mathrm{v}}^{2}=\frac{1}{\omega} \sum_{t=1}^{\omega}(\hat{\sigma}(t)-\langle\sigma\rangle)^{2}
$$

Table IV. - Computed values of the parameters related to the fitted periodic component in the standard deviation; $\langle\sigma\rangle=$ mean value of the 365 daily standard deviations $\sigma(t) ; D_{1}$ and $E_{1}=$ Fourier coefficients of the first harmonic; $P_{\mathrm{v}}=$ contribution of the first harmonic to the variance $\hat{\sigma}_{\mathrm{v}}^{2} ; \hat{\sigma}_{\mathrm{v}}^{2}=$ variance of the 365 daily standard deviations $\hat{\sigma}(t)$.

$\begin{array}{llllll}\text { Station } & \langle\sigma\rangle & D_{1} & E_{1} & P_{\mathrm{a}} & \hat{\sigma}_{\mathrm{v}}^{2} \\ & \left({ }^{\circ} \mathrm{C}\right) & \left({ }^{\circ} \mathrm{C}\right) & \left({ }^{\circ} \mathrm{C}\right) & (\% & \left({ }^{\circ} \mathrm{C}\right)\end{array}$

\begin{tabular}{lccccc}
\hline Alghero & 2.1 & 0.25 & -0.02 & 20.2 & 0.15 \\
\hline Ancona & 2.5 & 0.34 & 0.22 & 36.2 & 0.23
\end{tabular}

\begin{tabular}{lccccc}
\hline Napoli & 2.3 & 0.55 & 0.17 & 55.2 & 0.30 \\
\hline Torino & 2.6 & 0.24 & 0.26 & 31.3 & 0.20 \\
\hline Vigna di Valle & 2.4 & 0.09 & 0.19 & 13.2 & 0.17
\end{tabular}

Figure 5 shows the fitted function, $\sigma(t)$, and the 365 computed standard deviations $\hat{\sigma}(t)$ for the Torino station.

\section{Simulated daily ambient temperature data.}

In the previous section we have shown that a good model drawn for the empirical sequences of daily ambient temperature, $T(i, t)$, is given by equation (6) with $\mu(t)$ and $\sigma(t)$ given respectively by equation (17) and equation (18) and $X(i, t)$ given by equation (5).

On the basis of these results, simulated values can be obtained according to the following procedure :

a) generate a series of $n \cdot \omega$ random numbers $Y(i, t)$, with normal distribution, zero mean and standard deviation equal to $\left(1-\rho^{2}\right)^{1 / 2}$, with $\rho=0.78$;

b) use the relation (5), always with $\rho=0.78$, to construct à sequence of $n \cdot \omega$ number with zero mean, unit standard deviation and normal distribution;

c) obtain the daily ambient temperature from equation (6) with $\mu(t)$ and $\sigma(t)$ given respectively by equations (17) and (18).

Using the above procedure we have computed 7300 simulated values of $T(i, t)$ for each one of the examined localities.

In table $\mathrm{V}$ we show mean and standard deviation of the simulated and experimental series $T(i, t)$, while while in figure 6 we show the histograms of the simulated data and of the experimental data for the Torino station.
Table V. - Mean and variance of the experimental and simulated values of the daily ambient temperature.

\begin{tabular}{|c|c|c|c|c|}
\hline \multirow[b]{2}{*}{ Station } & \multicolumn{2}{|c|}{ Experimental data } & \multicolumn{2}{|c|}{ Generated data } \\
\hline & $\begin{array}{c}\text { Mean } \\
\left({ }^{\circ} \mathrm{C}\right)\end{array}$ & $\begin{array}{l}\text { Variance } \\
\left({ }^{\circ} \mathrm{C}^{2}\right)\end{array}$ & $\begin{array}{l}\text { Mean } \\
\left({ }^{\circ} \mathrm{C}\right)\end{array}$ & $\begin{array}{c}\text { Variance } \\
\left({ }^{\circ} \mathrm{C}^{2}\right)\end{array}$ \\
\hline Alghero & 16.2 & 29.5 & 16.2 & 29.0 \\
\hline Ancona & 14.6 & 48.5 & 14.6 & 47.6 \\
\hline Napoli & 15.2 & 35.7 & 15.1 & 35.1 \\
\hline Torino & 11.2 & 62.5 & 11.2 & 61.5 \\
\hline Vigna di Valle & 14.5 & 43.8 & 14.5 & 41.7 \\
\hline
\end{tabular}

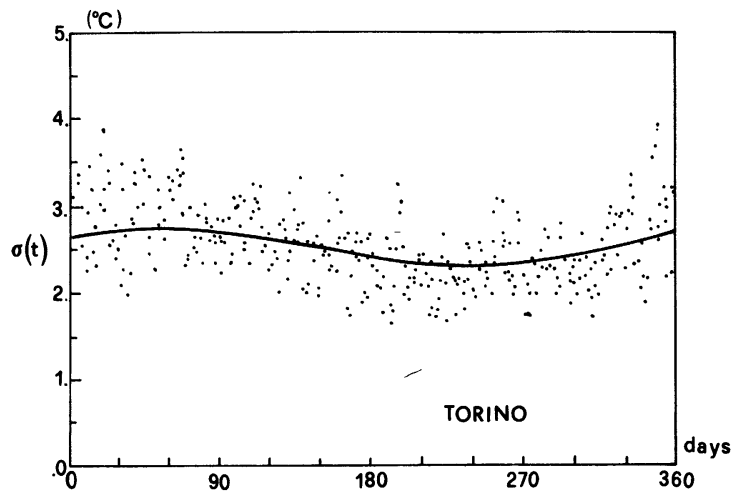

Fig. 6. - Torino station : $\sigma(t)$ vs. time (day of the year); continuous curve $=$ fitted function; dotted curve $=$ computed values $\hat{\sigma}(t)$.

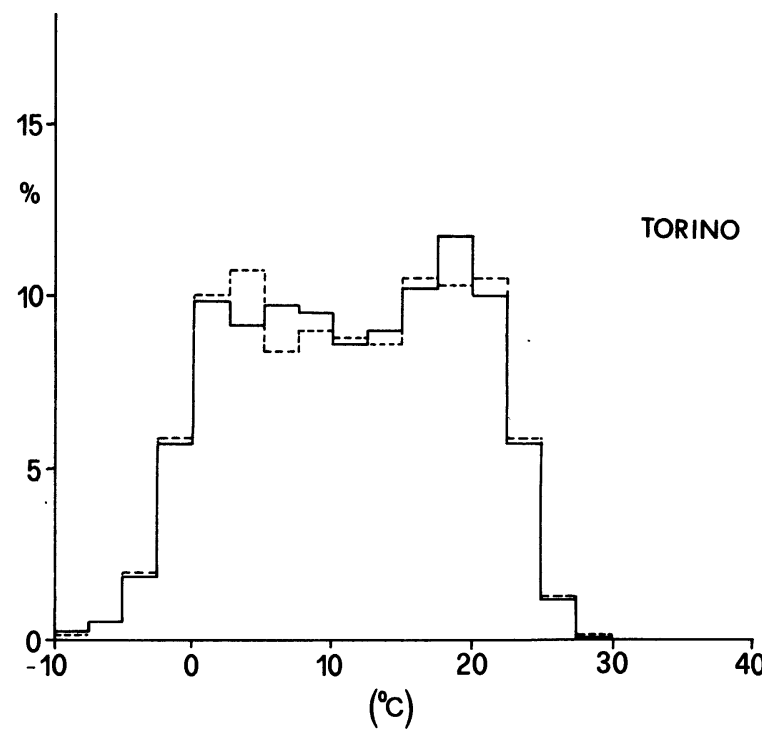

Fig. 7. - Torino station : frequency distribution of the daily ambient temperature. Comparison between experimental data (continuous step curve) and simulated data (dashed curve). 


\section{Conclusions.}

We have analysed the daily ambient temperature time series of 20 years of data from five stations in Italy.

We have shown that the time series can be described as :

$$
T(i, t)=\mu(t)+\sigma(t) \cdot X(i, t)
$$

where both $\mu(t)$ and $\sigma(t)$ are well fitted by the expansion in Fourier series (see section 5).

The series $X(i, t)$ is a dependent stochastic variable that follows the first-order autoregressive model of dependence (see section 4). The autocorrelation coefficient result quite independent on time and its variations with the locality are negligible (at least for Italian climate).

We have described a method which uses equation (5) and equation (6) to simulate daily ambient temperature values which have the same mean, variance and autocovariance as hystorical time series. The method requires the following parameters :

a) the average yearly value of $\mu(t)$ and $\sigma(t)$, i.e. $\langle\mu\rangle$ and $\langle\sigma\rangle$

b) the coefficient of the first harmonic of the development in Fourier series of both $\mu(t)$ and $\sigma(t)$;

c) the value of the first autocorrelation coefficient of the variable $X$;

d) the distribution of the variable $Y$.

The method, that requires only a small computing effort, can be used for the design and performance calculations of renewable energy technology (e.g. solar energy systems).

Furthermore the analysis gives some new evidence that, at least for the Mediterranean zone, Markovian models fit adequately to daily weather events (see Ref. [2-5, 9] for daily solar irradiance, and Ref. [16] for wind data).

\section{References}

[1] Bartoli, B., Catalanotti, S., Cuomo, V., Francesca, M., Serio, C., Silvestrini, V. and Troise, G., Statistical Correlation between Daily and Monthly Averages of Solar Radiation Data, Il Nuovo Cimento 2C 2 (1979) 222-234.

[2] Bartoli, B., Coluzzi, B., Cuomo, V., Francesca, M. and SERIO, C., Autocorrelation of Daily Global Solar Radiation, Il Nuovo Cimento 4C 2 (1981) 113-122.

[3] Amato, U., Andretta, A., Bartoli, B., Coluzzi, B., Cuomo, V., Fontana, F. and Serio, C., Markov processes and Fourier Analysis as a Tool to Describe and Simulate Daily Solar Irradiance, submitted to Solar Energy (1984).

[4] Bolleau, E., Discussion d'un Modèle Statistique en Météorologie Solaire, Revue Phys. Appl. 14 (1979) 145-152.

[5] Guerrier, B., Boileau, E. and Benard, C., Analyse Statistique Temporelle de l'Irradiation Solaire Globale: Modélisation d'une variable réduite à l'aide de Modèles stochastiques A.R.M.A., Revue Phys. Appl. 15 (1980) 93-102.

[6] Nichs, A. D. and HARP, J. F., Stochastic Generation of Temperature and Solar Radiation Data, J. Hydrology 48 (1980) 1-17.

[7] Lestienne, R., Application du Modèle Markovien simplifié à l'étude du Comportement du Stockage d'une Centrale Solaire, Revue Phys. Appl. 14 (1979) 139-144.
[8] Solcedo, A. C. and Recio, J. M. B., Fourier Analysis of meteorological data to obtain a typical annual time function, Solar Energy, 32, No. 4 (1984) 479488.

[9] BoILEAU, E., Use of some simple statistical models in solar meteorology, Solar Energy 30, No. 4 (1983) 333-339.

[10] BRINKWORTH, B. J., Autocorrelation and stochastic modeling of Sequences, Solar Energy 19 (1977) 343349.

[11] Aeronautica Militare Italiana : dati delle rete attinometrica.

[12] Jenkins, G. M., WATTS, D. G., Spectral analysis and its applications (Holden Day, S. Francisco - USA) 1968.

[13] Yevjevich, V., Stochastic processes in Hydrology (Water Resources Publications, Fort Collins, Colorado-USA) 1972.

[14] SCHEFFE, H., The analysis of variance (J. Wiley \& Sons, New York, USA) 1959.

[15] Kendall, M., Stuart, A., The advanced theory of statistics (Charles Griffin \& Co Ltd., London) 1976.

[16] Canas, J. R., Guzman, A. G. and Villasante, A. L., A stochastic model for wind occurrence, J. Appl. Meteor. 21 (1982) 740-744. 